\title{
Bioinspired Fog Collection Surface of Conical Brass With Gradient Wettability
}

\section{yanling wan ( $\square$ wanyl@cust.edu.cn )}

Changchun University of Science and Technology https://orcid.org/0000-0002-1320-5094

\section{Caiyun Zhang}

Changchun University of Science and Technology

\section{Pu Cui}

Changchun University of Science and Technology

Huadong Yu

Changchun University of Science and Technology

\section{Research Article}

Keywords: Pine needle, Fog harvesting, Biomimetic, Functional, Microstructure

Posted Date: April 6th, 2021

DOI: https://doi.org/10.21203/rs.3.rs-389372/v1

License: (9) This work is licensed under a Creative Commons Attribution 4.0 International License. Read Full License 


\title{
Bioinspired fog collection surface of conical brass with gradient
}

\section{wettability}

\author{
Yanling Wan*, Caiyun Zhang, Pu Cui, Huadong Yu \\ Key Laboratory of Cross-scale Micro and Nano Manufacturing (Changchun University of Science and \\ Technology), Ministry of Education, Changchun 130000, China
}

\begin{abstract}
How to obtain more fresh water from nature economically and efficiently is a hot issue that needs to be solved urgently. Herein, inspired by the directional fog collection ability of the surface of pine needles, a wettability-gradient-brass-cone (WGBC) was prepared through a two-step process of precision cutting and chemical etching, producing a surface with functions of fog collection and droplet movement to the root of the cone. On the other hand, through experimental exploration and optimization of taper parameters, the excellent directional collection velocity of droplets was obtained. This work may direct the design of gradient wetting surfaces by mimicking the structure of pine needles and explore potential applications in fog harvesting.
\end{abstract}

Keywords: Pine needle; Fog harvesting; Biomimetic; Functional; Microstructure

\section{Introduction}

How to obtain fresh water efficiently and cheaply has become a worldwide problem, and is also one of the most important measures to realize the sustainable development of human society [1-4]. Collecting water directly from fog is suggested as an excellent solution to meet the demand for fresh water partially, and potentially totally in specific areas of high frequency of fog events. [5]. Many plants and animals in nature have evolved unique directional water harvesting abilities, such as cicada, cacti, and desert beetles[6-8]. The unique microstructure and special wettability on the biological surface 
are the key to achieving directional fog collection. As a matter of fact, a number of efficient technologies have currently emerged for preparing bioinspired artificial surfaces $[9,10]$. This active water harvesting surface, which does not require any external energy input, holds great promise for applications to alleviate water scarcity.

However, it is still a challenge for fabricating a surface that enables both fast deposition and directional transport simultaneously. In our previous study on pine needles, it was found that its surface had a unique function of fog collection and transport of liquid droplets in a directional manner. The shape of the pine needle and the microscopic topography of the surface are the key to achieving the mist collection function. Inspired by the fog-collection principle of pine needles, therefore, a wettability-gradient-brasscone (WGBC) was fabricated by chemical etching and we hope to expand the field of fog directional collection and provide a theoretical basis for the design and preparation of bionic structures through this research.

\section{Experimental section}

\subsection{Materials}

The material used in this paper is the brass rod with a diameter of $6 \mathrm{~mm}(\mathrm{H} 62, \mathrm{Wu}$ Hook Metal Co, China). Experimental reagents include $(\mathrm{NH} 4)_{2} \mathrm{~S}_{2} \mathrm{O}_{8}$ (Beijing Bailingwei Technology Co, China), deionized water (Jilin Haodi Chemical Reagent Distribution Co, China), $\mathrm{NaOH}$ and $\mathrm{C}_{2} \mathrm{H}_{5} \mathrm{OH}$ (Beijing Chemical Plant, China), and all of them are analytical reagents.

\subsection{Fabrication}

Copper rods are machined into cones of different tapers with a length of $3 \mathrm{~cm}$ by the microfabrication lathe (The positioning accuracy of the microfabrication lathe is $2 \mu \mathrm{m}$, and the re-orientation accuracy is $0.5 \mu \mathrm{m}$. Processing parameters are as follows: spindle speed 
$\mathrm{n}=2000 \mathrm{r} / \mathrm{min}$, feed rate $\mathrm{V}_{\mathrm{f}}=500 \mathrm{~mm} / \mathrm{min}$, cutting depth $\mathrm{a}_{\mathrm{p}}=30 \mu \mathrm{m}$, and the actual taper machining error is about \pm 0.4$)$. The copper cone is polished by \#400, \#800, \#1200, \#2000 sandpaper, respectively, and then rinsed with ethanol and deionized water.

The preparation of the wetting gradient was obtained by chemical etching. Step 1: a solution was prepared by mixing, $2.67 \mathrm{~mol} / \mathrm{L}$ of $\mathrm{NaOH}$ solution with $0.15 \mathrm{~mol} / \mathrm{L}$ of $(\mathrm{NH} 4)_{2} \mathrm{~S}_{2} \mathrm{O}_{8}$ solution. Step 2: the treated conical brass was corroded by three sections for corrosion. Specifically, the brass cone was clamped securely with the tip facing upwards and immersed in the mixed solution for $1 \mathrm{~cm}$ from the $\operatorname{root}($ fig. $1 . \mathrm{h})$, and then rinsed $1 \mathrm{~cm}$ every 30 minutes until the tip was immersed. Finally, the gradient wetted brass cone (GWBC) was obtained.

\subsection{Measurement and characterization}

Scanning electron-microscopy (SEM, Zeiss, EVO MA25, Germany) was used to observe the microstructure of the specimen. And X-ray energy dispersive spectroscopy was used to analyze the elemental changes on the surface of the material (EDS, Oxford Instruments, England). Fog harvesting mechanism of GWBC was examined using saturated fog flow (by an ultrasonic humidifier with a fog flow of $35 \mathrm{ml} / \mathrm{h}$ ). A CCD camera (25mm, 1:1.4, Computar) was employed to monitor the whole process of capturing fog in the experiment. The optical contact angle meter system was used to measure the contact angle. Environmental temperature was $22 \pm 3^{\circ} \mathrm{C}$, and relative humidity was $55 \%-65 \%$.

\section{Results and discussion}

\subsection{Wettability and surface morphology}

We know that the deposition efficiency of water droplets on different wettability surfaces is different, and the hydrophilic surface has a higher mist collection efficiency [11]. With this fundamental understanding, we used chemical etching to generate 
hydrophilic copper hydroxide nanostructures on the surface, thereby achieving improved mist collection capabilities.

SEM observation of WGBC at different locations (with different corrosion times) revealed that there were some bamboo leaf-like microstructures on the surface. As the reaction time increased, the structure gradually increased (Fig.1.a-c).
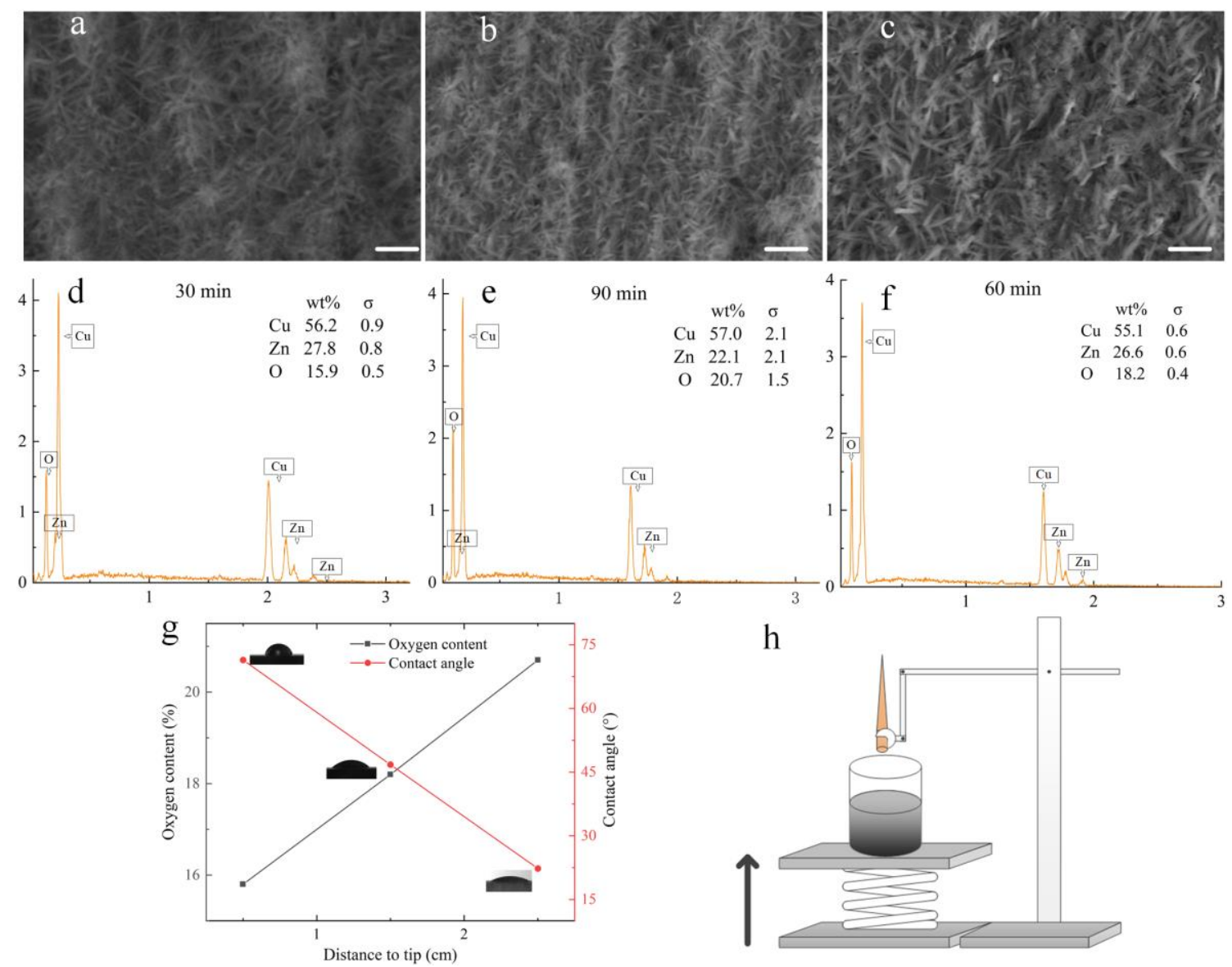

Fig.1. (a-b) SEM images of the GWBC, and (d-f) EDS diagram of GWBC surface, showed the sections where the distance to the tip is $0.5 \mathrm{~cm}, 1.5 \mathrm{~cm}$ and $2.5 \mathrm{~cm}$, respectively. Scale bar, $10 \mu \mathrm{m}$. (g) Composite line graph of oxygen content and contact angle. (h) Schematic diagram of GWBC preparation.

The EDS patterns obtained for the GWBC were compared, as shown in Fig.1.(d-f). The content of copper and zinc elements on the surface decreased with increasing corrosion time, while the oxygen content rose rapidly. When brass is put into the mixed solution, the following chemical reaction occurs: 
$\mathrm{Cu}+4 \mathrm{NaOH}+\left(\mathrm{NH}_{4}\right)_{2} \mathrm{~S}_{2} \mathrm{O}_{8} \rightarrow \mathrm{Cu}(\mathrm{OH})_{2}+2 \mathrm{Na}_{2} \mathrm{SO}_{4}+2 \mathrm{NH}_{3}+2 \mathrm{H}_{2} \mathrm{O}$

As the corrosion time increased, the $\mathrm{Cu}(\mathrm{OH})_{2}$ precipitation generated by the reaction on its surface increased, exhibiting hydrophilic property. The contact angle of the untreated copper cone surface was $82.3^{\circ}(4 \mu$ droplet). The contact angle decreased gradually from the root to the tip after the reaction of (1), so that a wetting gradient was formed on the surface.

\subsection{Measurement of the fog collection efficiency}

A key factor in the ability of the pine needle to move droplets in a directional manner is its conical structure [12]. To investigate the effect of cone taper on the water collection velocity, the droplet collection capacity of GWBC with different tapers was measured (Fig.2). Results indicated that the droplets captured on the surface of each cone, and the droplets captured at the tip moved towards the root and engulfed the water droplets along their path under the effect of the shape gradient. Meanwhile, an obvious transition occurred approximately every $1 \mathrm{~cm}$. The wetting gradient promoted the movement of droplets toward the root, resulting in directional collection. With the increase of the taper, the velocity of the droplet showed a trend of first increasing and then decreasing. When the taper was $6^{\circ}$, an optimal velocity was obtained for directional water collection of WGBC, with an average movement velocity of $0.058 \mathrm{~cm} / \mathrm{s}$. 


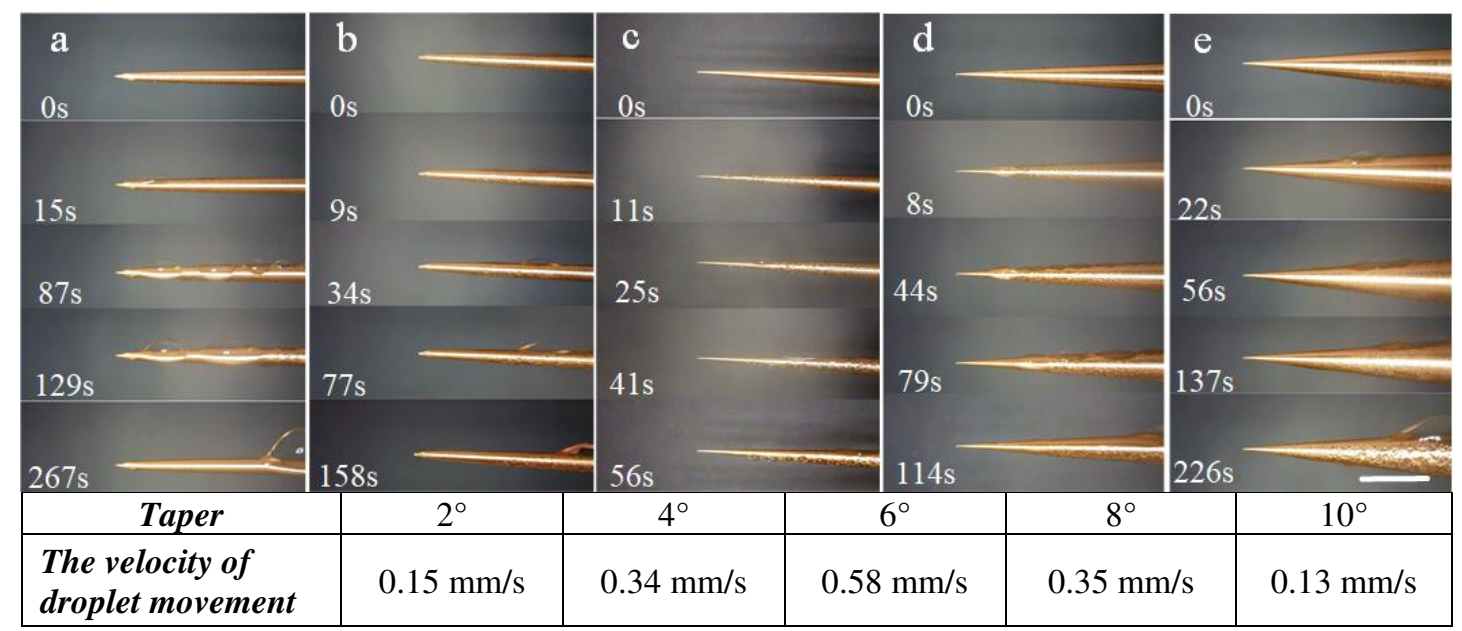

Fig.2. Fog collection performance testing of WGBC with different tapers. Figure a-e are experimental images of the directional fog collection of WGBC with tapers of $2,4,6,8$, and $10^{\circ}$, respectively. Scale bar, $1 \mathrm{~mm}$. The table below the image shows the corresponding the velocity of droplet movement.

Assuming that the droplet is elliptical on the surface of the cone, the droplet is mainly affected by surface adhesion $\mathrm{F}_{\mathrm{N}}$, Laplace pressure $\mathrm{F}_{\mathrm{L}}$ and gravity $\mathrm{F}_{\mathrm{G}}$, where the adhesion force of the droplet on the cone surface is[13]:

$$
F_{N}=\gamma_{L V} k \pi d / 2
$$

where $\gamma_{L V}$ stands for the surface tension of water. $k$ denotes the numerical factor considering the shape of the contact line and the variation of the contact angle along the contact line[13]. $\pi d / 2$ is the length of the contact line between the water droplet and the cone, and for simplicity of calculation, the contact line is considered to be the radius of the profile. The Laplace pressure[14] difference experienced by the droplet is:

$$
F_{L}^{\prime}=-\int_{r_{2}}^{r_{1}} \frac{2 \gamma_{L V}}{\left(r+R_{0}\right)^{2}} \cdot \sin \beta d z
$$

where $r$ is a radius variable, $r_{1}, r_{2}$ are respectively the radius of the section circle at both ends of the droplet on the cone. $R_{0}$ represents the radius of the water droplet. $\beta$ is the half apex-angle of section triangle and $z$ is an integrating variable. 
a

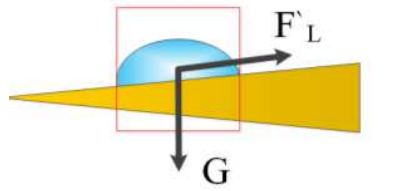

b

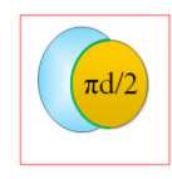

Fig.3. (a) Force analysis of WGBC placed horizontally. (b) is a schematic diagram of the water droplet contact line on the cone section.

When the cone taper is relatively small $\left(1-2^{\circ}\right)$, the captured droplet was capable of moving towards the root if the adhesion force between the droplet and the surface could be overcome under the drive of Laplace pressure. At this time, the $F_{L}{ }_{L}$ on both sides of the droplet was relatively small, resulting in a lower movement velocity of directional collection. And as the taper increases, the curvature difference between the two sides of the droplet gradually increased, making $F_{L}^{\prime}$ larger and thus increasing the droplet orientation movement velocity. However, if the taper exceeded the limit allowed, the effect of gravity became greater, which was not conducive to the directional movement of the droplets [15]. This is the reason why the velocity of droplets moving on the GWBC surface increased first and then decreased with the taper.

\section{Conclusions}

In summary, inspired by the cone structure of pine needles a directional fog collection surface is developed successfully. The brass cones with different tapers were prepared by micro-cutting, and then the gradient wettability was obtained by different chemical etching reaction times, finally the WGBCs were prepared.

In the experiment, (1) as the mixed solution of $\mathrm{Cu}(\mathrm{OH})_{2}$ and $(\mathrm{NH} 4)_{2} \mathrm{~S}_{2} \mathrm{O}_{8}$ corrodes the brass surface, a hydrophilic copper hydroxide film is formed. The surface changes from hydrophobic to hydrophilic, resulting in a better fog collection capacity. (2) When the WGBC is placed horizontally, the Laplace pressure is the dominant force, allowing the droplets coalescing at the tip of the cone to migrate directionally. The WGBC with a 
taper of $6^{\circ}$ has an excellent performance in directional water collection, with an average movement velocity of $0.058 \mathrm{~cm} / \mathrm{s}$. Inspired by pine needles, this research provides a new idea for the directional fog collection and contributes to future research and applications in fog harvesting.

\section{References}

[1] M.M. Mekonnen, A.Y. Hoekstra, Four billion people facing severe water scarcity, Science Advances 2(2) (2016) e1500323-e1500323.

[2] A.K. Mishra, V.P. Singh, Drought modeling-A review, Journal of Hydrology 403(1-2) (2011) 157175.

[3] A. Pa, Y.H. Gin, Y.C. Lin, M. Reinhard, Impacts of emerging organic contaminants on freshwater resources: Review of recent occurrences, sources, fate and effects, Science of the Total Environment $408(24)(2010) 6062-6069$.

[4] S. Ngcobo, G. Jewitt, S.I. Stuart-Hill, M.L. Warburton, Impacts of global change on southern African water resources systems, Current Opinion in Environmental Sustainability 5(6) (2013) 655-666.

[5] O. Klemm, R.S. Schemenauer, A. Lummerich, P. Cereceda, V. Marzol, D. Corell, J.V. Heerden, D. Reinhard, T. Gherezghiher, J. Olivier, Fog as a fresh-water resource: overview and perspectives, Ambio 41(3) (2012) 221-234.

[6] H. Xie, H.X. Huang, H.Y. Mi, Gradient Wetting State for Droplet Transportation and Efficient Fog Harvest on Nanopillared Cicada Wing Surface, Materials Letters 221 (2018) 123-127.

[7] Z.-X. Huang, X. Liu, J. Wu, S.-C. Wong, J.-P. Qu, Electrospinning water harvesters inspired by spider silk and beetle, Materials Letters 211 (2018) 28-31.

[8] J. Ju, H. Bai, Y. Zheng, T. Zhao, R. Fang, L. Jiang, A multi-structural and multi-functional integrated fog collection system in cactus, Nature Communications.

[9] R.K. Upadhyay, P.R. Waghmare, Green Preparation of Copper Surfaces with Wettability Contrast for Guided Fluid Transport and Fog Harvesting Application, Materials Letters 246(JUL.1) (2019) 223-226.

[10] Y. Xing, W. Shang, Q. Wang, S. Feng, Y. Hou, Y. Zheng. Integrative Bioinspired Surface with Wettable Patterns and Gradient for Enhancement of Fog Collection. ACS applied materials \& interfaces,2019, 
11(11): 10951-10958.

[11] Dai X, Nan S, Nielsen S O, et al. Hydrophilic directional slippery rough surfaces for water harvesting[J]. Adv, 2018, 4(3).

[12] Y. Wan, P. Cui, J. Xu, H. Yu, Directional water-collecting behavior of pine needle surface, Materials Letters 255(Nov.15) (2019) 126561.1-126561.4.

[13] Y. Jiang, C. Machado, S. Savarirayan, N.A. Patankar, K.C. Park, Onset time of fog collection, Soft Matter 15 (2019).

[14] L. Lorenceau, D. Qur, Drops on a conical wire, Journal of Fluid Mechanics 510(510) (2004) 29-45.

[15] T. Xu, Y. Lin, M. Zhang, W. Shi, Y. Zheng, High-Efficiency Fog Collector: Water Unidirectional Transport on Heterogeneous Rough Conical Wires, Acs Nano (2016) acsnano.6b05595. 
Figures
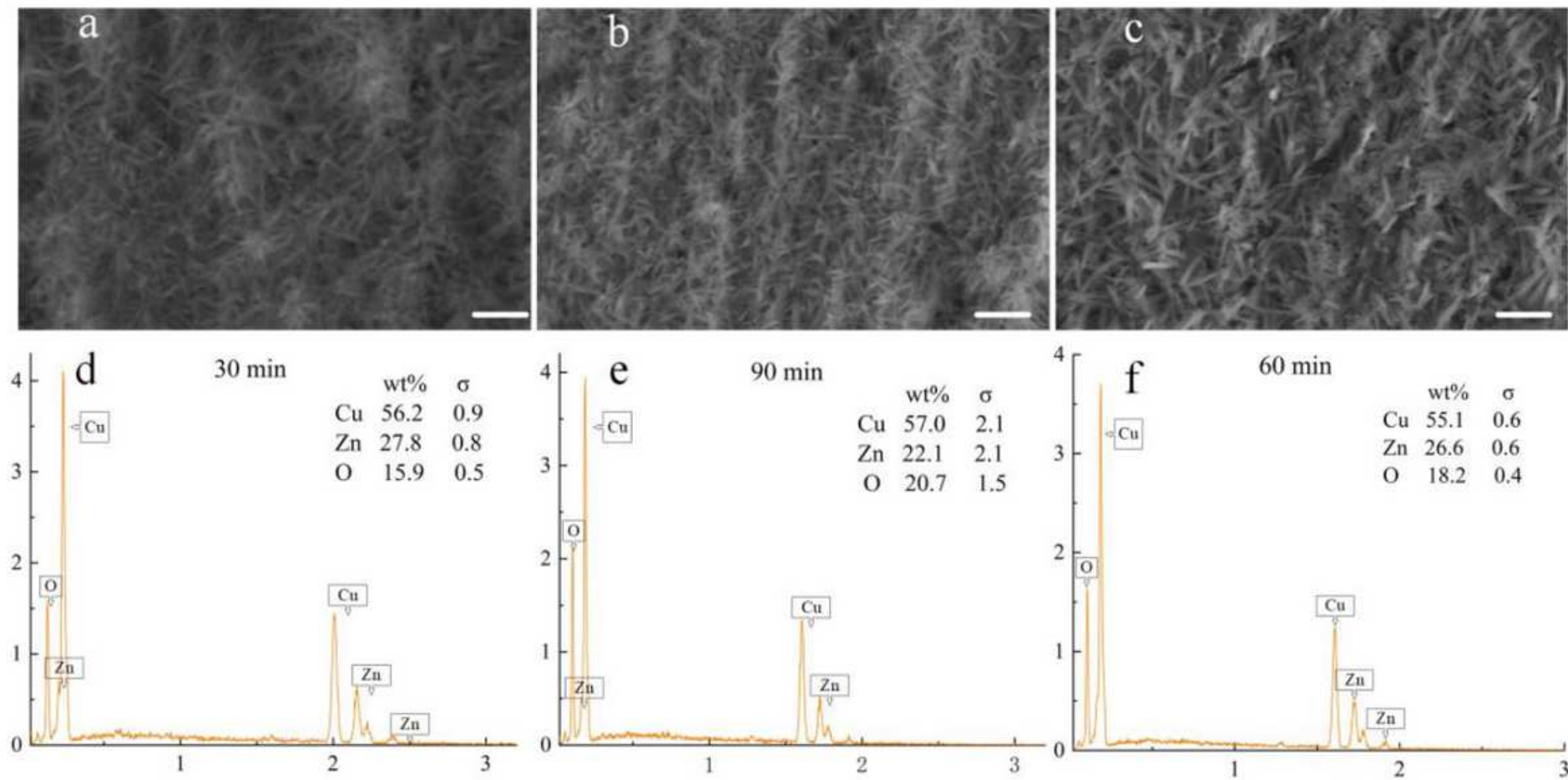

${ }^{4}[\mathrm{f}$

$60 \mathrm{~min}$

$\mathrm{wt} \% \quad \sigma$

$\mathrm{Cu} 55.1 \quad 0.6$

$\mathrm{Zn} \quad 26.6 \quad 0.6$

O $\quad 18.2 \quad 0.4$
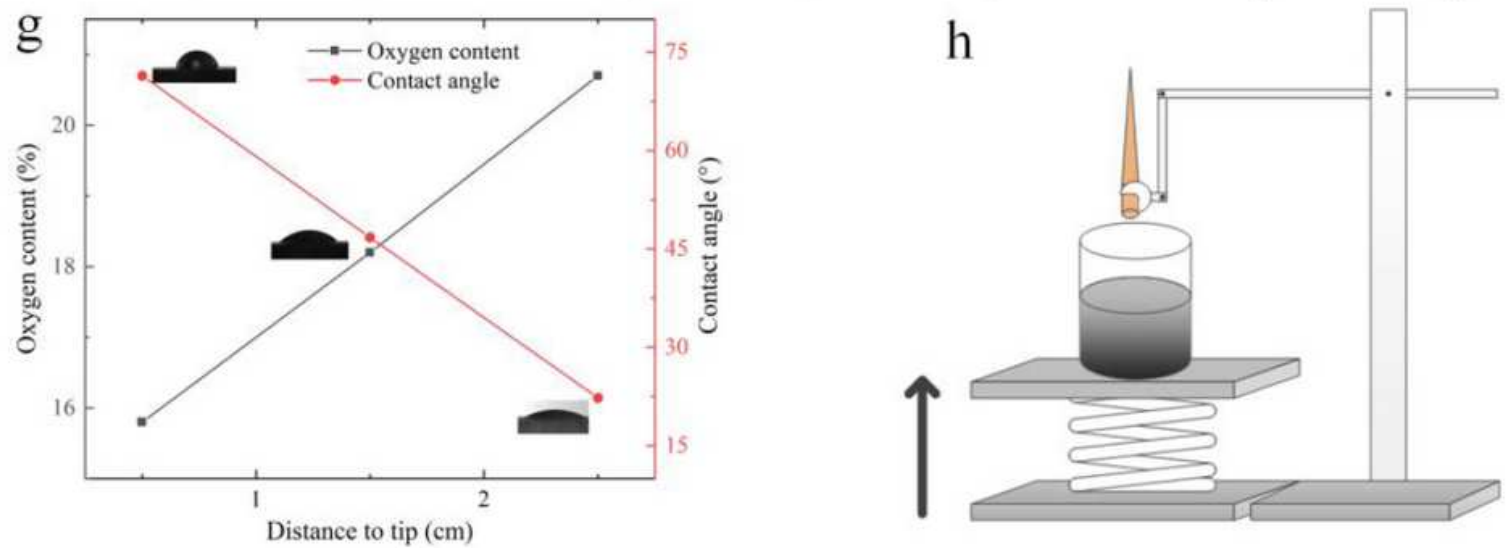

\section{Figure 1}

(a-b) SEM images of the GWBC, and (d-f) EDS diagram of GWBC surface, showed the sections where the distance to the tip is $0.5 \mathrm{~cm}, 1.5 \mathrm{~cm}$ and $2.5 \mathrm{~cm}$, respectively. Scale bar, $10 \mu \mathrm{m}$. $(\mathrm{g})$ Composite line graph of oxygen content and contact angle. (h) Schematic diagram of GWBC preparation. 


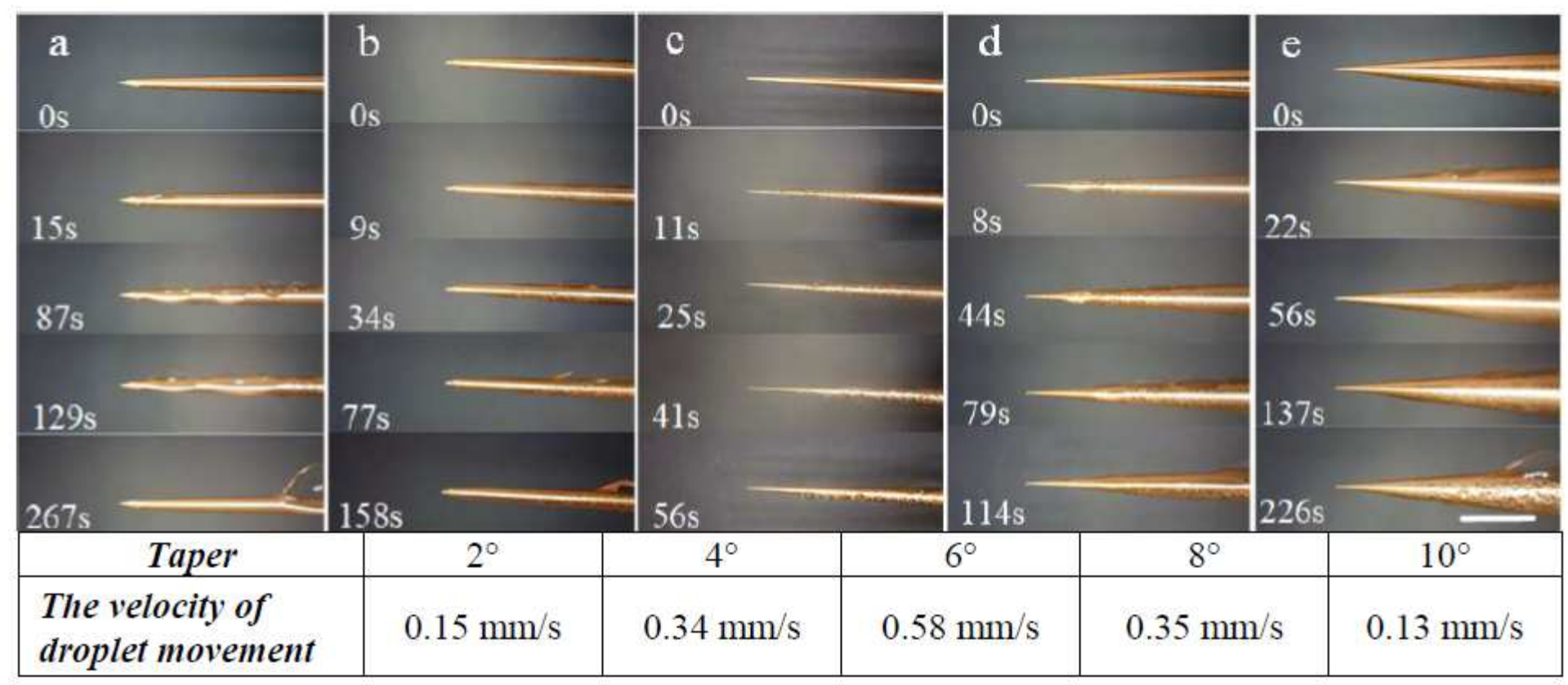

\section{Figure 2}

Fog collection performance testing of WGBC with different tapers. Figure a-e are experimental images of the directional fog collection of WGBC with tapers of $2,4,6,8$, and $10^{\circ}$, respectively. Scale bar, $1 \mathrm{~mm}$. The table below the image shows the corresponding the velocity of droplet movement.

a
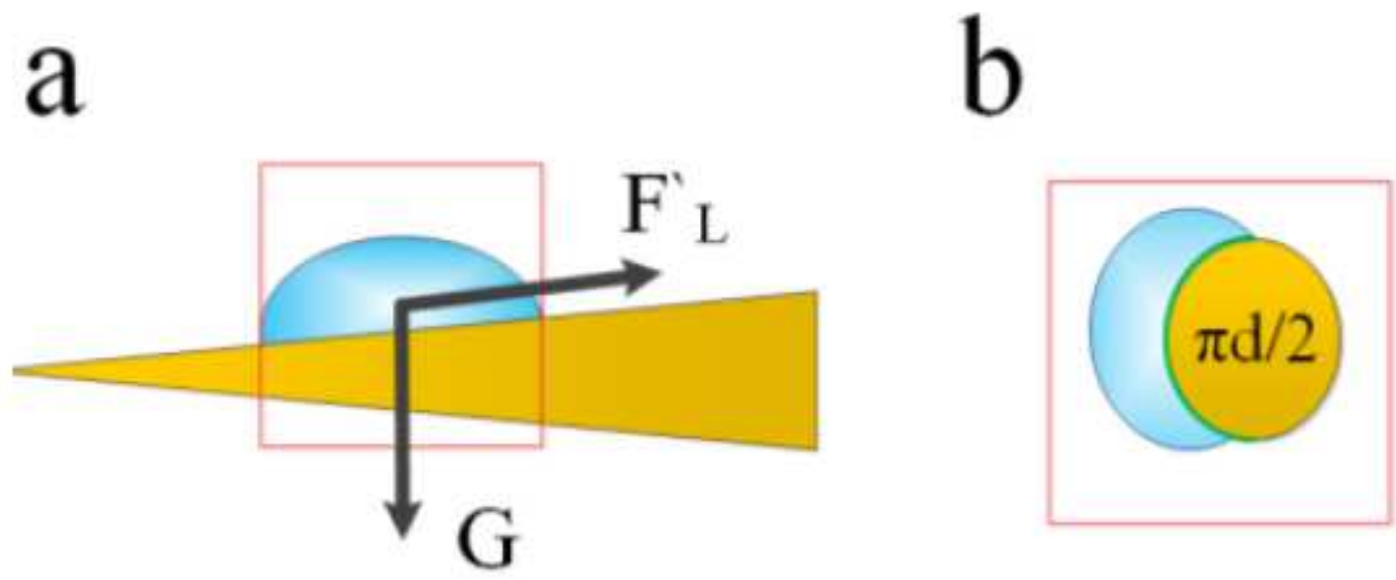

Figure 3

(a) Force analysis of WGBC placed horizontally. (b) is a schematic diagram of the water droplet contact line on the cone section. 